\title{
Second-Order Expected Utility
}

\author{
Simon Grant* $\quad$ Ben Polak ${ }^{\dagger} \quad$ Tomasz Strzalecki ${ }^{\ddagger}$ \\ Preliminary version: November 2009
}

\begin{abstract}
We present two axiomatizations of the Second-Order Expected Utility model in the context of the standard models of choice under uncertainty.
\end{abstract}

\section{Introduction}

Second-Order Expected Utility (SOEU) is a very simple model of preferences that is consistent with Ellsberg-type behavior and more generally allows for sensitivity to the source of uncertainty. It was first axiomatized in the Anscombe-Aumann setting by Neilson $(1993,2009)$ and was later studied by Nau $(2001,2006)$ and Ergin and Gul (2009) in the context of a Savage model with a product state space; see also Chew and Sagi (2008) for a general study of source sensitivity and Abdellaoui, Baillon, Placido, and Wakker (2009) for an experimental investigation.

The SOEU preferences intersect with standard models. As was shown by Strzalecki (2009), the class of multiplier preferences of Hansen and Sargent (2001) is precisely the intersection of the SOEU preferences and the variational preferences of Maccheroni, Marinacci, and Rustichini (2006). In addition, SOEU can be thought of as a special case of the model of Klibanoff, Marinacci, and Mukerji (2005) (in which the support of measure $\mu$ consists of Dirac measures on $S$ ).

\footnotetext{
*Department of Economics, Rice University

${ }^{\dagger}$ Department of Economics \& School of Management, Yale University

${ }^{\ddagger}$ Department of Economics, Harvard University
} 
In this note we present two axiomatizations that show how to obtain SOEU in standard models. The first extends and relies on a result of Werner (2005) and says that any preferences that satisfy our basic technical axioms and are ambiguityaverse in the sense of Ghirardato and Marinacci (2002) and also satisfy the Savage's Sure Thing Principle are SOEU. This is because GM-ambiguity aversion is related to "translation invariance along the certainty line", which together with the Sure Thing Principle plays the role of state independence and implies SOEU. The second result imposes a version of "translation invariance along the certainty line" directly. We are using an axiom that is very weak by itself, but in the presence of Uncertainty Aversion of Schmeidler (1989) and the Sure Thing Principle delivers SOEU.

\section{Preliminaries}

\section{$2.1 \quad$ Setup}

Let $S$ be a finite state space. Let $\Delta(Z)$ be the set of simple probability measures on a set of prizes $Z$. Acts are mappings $f: S \rightarrow \Delta(Z)$; let $\mathcal{F}$ denote the set of acts. If $f, g \in \mathcal{F}$ and $E \subseteq S$, then $f_{E} g$ denotes an act with $f_{E} g(s)=f(s)$ if $s \in E$ and $f_{E} g(s)=g(s)$ if $s \notin E$. Let $\Delta(S)$ be the set of probability measures on $S$. For any

$p \in \Delta(S)$ and any $\xi \in \mathbb{R}^{S}$ let $\mathbb{E}_{p} \xi:=\sum_{s \in S} \xi(s) p(s)$. For any $p \in \Delta(S)$ and any act $f \in \mathcal{F}$ let $\mathbb{E}_{p} f$ denote the constant act with $\mathbb{E}_{p} f(z)=\sum_{s \in S} f(s)(z) p(s)$, i.e., the probability mixture of the lotteries that obtain in different states with weights given by $p$.

Every act $f: S \rightarrow \Delta(Z)$ involves two sources of uncertainty: first, the payoff of $f$ is contingent on the state, for which there is no objective probability given; second, given the state, $f(s)$ is an objective lottery. The existence of two sources of uncertainty enables a distinction between purely objective lotteries, i.e., acts which pay the same lottery $\pi \in \Delta(Z)$ irrespectively of the state of the world and purely subjective acts, i.e., acts that in each state of the world pay off a degenerate lottery $\delta_{z}$ for some $z \in Z$, which possibly depends on $s$. With the usual abuse of notation, let $\Delta(Z)$ denote the set of purely objective lotteries. Note that given $p \in \Delta(S)$ each purely subjective act $f$ induces the purely objective lottery $\mathbb{E}_{p} f \in \Delta(Z)$. 


\subsection{Anscombe-Aumann Expected Utility}

The standard Anscombe-Aumann expected utility model ranks acts according to

$$
f \mapsto \sum_{s \in S} u(f(s)) p(s)
$$

where $u: \Delta(Z) \rightarrow \mathbb{R}$ is an affine function and $p \in \Delta(S)$. This model imposes a uniform decision attitude towards objective risk and subjective uncertainty. To see this, observe that for any two purely objective lotteries $\pi^{\prime} \succsim \pi$ if and only if

$$
\sum_{z \in Z} u(z) \pi^{\prime}(z) \geq \sum_{z \in Z} u(z) \pi(z)
$$

and for any two purely subjective acts $f^{\prime} \succsim f$ if and only if

$$
\sum_{z \in Z} u(z) \mathbb{E}_{p} f^{\prime}(z) \geq \sum_{z \in Z} u(z) \mathbb{E}_{p} f(z)
$$

In particular, any purely subjective act $f$ is indifferent to the objective lottery $\mathbb{E}_{p} f$ that it induces.

\section{$2.3 \quad$ Second-Order Expected Utility}

The SOEU model ranks acts according to

$$
f \mapsto \sum_{s \in S} \phi(u(f(s))) p(s)
$$

where $u: \Delta(Z) \rightarrow \mathbb{R}$ is an affine function with range $\mathcal{U}, \phi: \mathcal{U} \rightarrow \mathbb{R}$ is a strictly increasing function, and $p \in \Delta(S)$. By contrast to the Anscombe-Aumann expected utility model, this model allows the decision maker to have different attitudes towards objective risk and subjective uncertainty. To see that, observe that for any two purely objective lotteries $\pi^{\prime} \succsim \pi$ if and only if

$$
\sum_{z \in Z} u(z) \pi^{\prime}(z) \geq \sum_{z \in Z} u(z) \pi(z)
$$


On the other hand, for any two purely subjective acts $f^{\prime} \succsim f$ if and only if

$$
\sum_{z \in Z} \phi(u(z)) \mathbb{E}_{p} f^{\prime}(z) \geq \sum_{z \in Z} \phi(u(z)) \mathbb{E}_{p} f(z)
$$

These preferences are different to the extent to which the function $\phi$ is nonlinear. In particular, if $\phi$ is concave, any purely subjective act $f$ is ranked lower than the objective lottery $\mathbb{E}_{p} f$ that it induces.

\subsection{Basic Axioms}

Axiom A.1 (Weak Order) The relation $\succsim$ is complete, and transitive.

Axiom A.2 (Independence on constant acts) For all $\pi, \rho, \sigma \in \Delta(Z)$ and $\alpha \in$ $(0,1): \pi \sim \rho$ implies $\alpha \pi+(1-\alpha) \sigma \sim \alpha \rho+(1-\alpha) \sigma$.

Axiom A.3 (Continuity) If $f, g, h, \in \mathcal{F}$ then sets $\{\alpha \in[0,1] \mid \alpha f+(1-\alpha) g \succsim h\}$ and $\{\alpha \in[0,1] \mid h \succsim \alpha f+(1-\alpha) g\}$ are closed.

Axiom A.4 (Monotonicity) If $f, g \in \mathcal{F}$ and $f(s) \succsim g(s)$ for all $s \in S$, then $f \succsim g$.

Axiom P2 (Sure Thing Principle) For all events $E \in \Sigma$ and acts $f, g, h, h^{\prime} \in$ $\mathcal{F}$ : if $f_{E} h \succsim g_{E} h$ then $f_{E} h^{\prime} \succsim g_{E} h^{\prime}$.

Definition 1. A state $s$ is non-null iff there exists $f \in \mathcal{F}$ and $\pi, \rho \in \Delta(Z)$ such that $\pi_{s} f \succ \rho_{s} f$.

\subsection{Canonical Representation}

In the present setting the canonical result of Debreu (1960) implies the following.

Theorem 1. Suppose there are at least three non-null states. The preference $\succsim$ satisfies axioms A.1-A.4 and P2 iff there exists a non-constant affine function $u$ : $\Delta(Z) \rightarrow \mathbb{R}$ with range $\mathcal{U}$ and continuous weakly increasing functions $v_{s}: \mathcal{U} \rightarrow \mathbb{R}$, at least three of them nonconstant, such that $\succsim$ is represented by

$$
f \mapsto \sum_{s \in S} v_{s}(u(f(s)))
$$




\section{Results}

\subsection{GM-ambiguity aversion}

Our first result relies on the assumption that preferences are ambiguity averse according to Ghirardato and Marinacci (2002).

Axiom A.5 (GM-Ambiguity Aversion) There exists $p \in \Delta(S)$ such that for all $f \in \mathcal{F}$ and $\pi \in \Delta(Z): f \succsim \pi$ implies $\mathbb{E}_{p} u(f) \geq u(\pi)$.

Most standard models satisfy this axiom, for example the Maxmin Expected Utility preferences of Gilboa and Schmeidler (1989) and more generally the variational preferences of Maccheroni et al. (2006) and confidence preferences of Chateauneuf and Faro (2009), as well as the smooth preferences of Klibanoff et al. (2005) (as long as the function $\phi$ is concave).

The next theorem shows that this assumption is enough to force all of the functions $v_{s}$ in representation (1) to be identical up to multiplication by nonnegative coefficients $p(s)$.

Theorem 2. Suppose there are at least three non-null states. The preference satisfy axioms $A .1-A .5$ and $P \mathscr{2}$ iff it is represented by

$$
f \mapsto \sum_{s \in S} \phi(u(f(s))) p(s)
$$

for some non-constant affine function $u: \Delta(Z) \rightarrow \mathbb{R}$ with range $\mathcal{U}$ and a continuous, concave, and strictly increasing function $\phi: \mathcal{U} \rightarrow \mathbb{R}$ and a probability measure $p \in \Delta(S)$ such that $p(s)>0$ iff $s$ is non-null. Moreover, in this case $p$ is the measure with respect to which $\succsim$ is GM-ambiguity averse. Furthermore, if $(u, \phi, p)$ and $\left(u^{\prime}, \phi^{\prime}, p^{\prime}\right)$ represent $\succsim$, then there exists $\alpha, A>0, \beta, B \in \mathbb{R}$ such that $p^{\prime}=p$, $u^{\prime}=\alpha u+\beta$, and $\phi^{\prime}(\alpha r+\beta)=A \phi(r)+B$ for all $r$ in range of $u$.

This theorem extends and relies on Werner's (2005) axiomatization of risk averse expected utility. The main idea behind it is that Axiom A.5 implies that indifference curves in the utility space (i.e. the induced preferences on $\mathcal{U}^{S}$ ) have common supporting hyperplanes along the "certainty line" (i.e., the set of constant vectors in $\mathcal{U}^{S}$ ). 


\subsection{Uncertainty Aversion}

In this section we study preferences that satisfy Axioms A1-A4, P2, and additionally the Uncertainty Aversion axiom of Schmeidler (1989).

Axiom A.6 (Uncertainty Aversion) If $f, g \in \mathcal{F}$ and $\alpha \in(0,1), f \sim g$ implies $\alpha f+(1-\alpha) g \succsim f$.

As is well known, Axioms A.5 and A.6 are not nested in the presence of Axioms A.1-A.4. Moreover, in the presence of Axioms A.1-A.4 and P2, Axiom A.5 implies A.6 (as a consequence of our Theorem 2). However, Axiom A.6 does not imply Axiom A.5, as the following example shows.

Example 1. Let $\mathcal{U}=(0, \infty)$ and let $v_{1}(k)=\log (k)$ and $v_{2}(k)=v_{3}(k)=\sqrt{k}$. It is immediate that the preference represented by $f \mapsto v_{1}(u(f(1)))+v_{2}(u(f((2))+$ $v_{3}(u(f(3))$ has three non-null states and satisfies Axioms A.1-A.4, A6 and P2. However, it does not satisfy Axiom A.5. (If it did, then by Theorem 2 the preference would be state-independent; and in particular the slopes of the indifference curves in the utility space would coincide along the "certainty line", i.e. for all constant utility acts. However, the slopes are clearly varying along certainty line.)

In order to obtain the SOEU preferences we need to add another axiom that ensures precisely the needed independence that in Section 3.1 was guaranteed by Axiom A.5. There are strong known axioms which guarantee that: The Weak Certainty Independence axiom of Maccheroni et al. (2006) ensures that the indifference curves in the utility space are parallel and hence the slope at the certainty line is constant. Similarly, the Worst Independence axiom of Chateauneuf and Faro (2009) ensures that the indifference curves in the utility space are radial expansions of each other, which also guarantees that the slope at the certainty line is constant. However, such conditions are too strong for our purposes, as they restrict the functional form of $\phi$. It follows from Strzalecki (2009) that Weak Certainty Independence forces $\phi$ to be in the class of exponential functions. Similarly, it can be shown that the Worst Independence axiom forces $\phi$ to be in the class of power functions.

An axiom weaker than both of the ones above that still guarantees SOEU is the Translation Invariance at Certainty axiom of Rigotti, Shannon, and Strzalecki 
(2008). This axiom imposes that the sets of supporting hyperplanes of the indifference curves the are the same everywhere along the certainty line. However, this condition is still stronger than the one needed (as it in particular implies axiom A.5.). The weaker condition that we describe below ensures that the supporting hyperplanes are equal along the certainty line whenever they are unique.

We are interested in a subset of constant acts at which preferences are locally approximated by Anscombe-Aumann Expected Utility preferences. For an agent with such preferences, there exists a measure $p \in \Delta(S)$ such that for all constant acts $\pi \in \Delta(Z)$ and acts $f \in \mathcal{F}: \mathbb{E}_{p} f \succ \pi$ implies $f \succ \pi$, and $\pi \succ \mathbb{E}_{p} f$ implies $\pi \succ f$. By analogy,

Definition 2 (Locally EU). We say that $\succsim$ is locally $E U$ at the constant act $\pi$ with respect to $p^{\pi} \in \Delta(S)$ if, for all acts $f: \mathbb{E}_{p^{\pi}} f \succ \pi$ implies there exists an $\bar{\alpha} \in(0,1]$ such that for all $\alpha \in(0, \bar{\alpha}], \alpha f+(1-\alpha) \pi \succ \pi$ and $\pi \succ \mathbb{E}_{p^{\pi}} f$ implies there exists an $\bar{\alpha} \in(0,1]$ such that for all $\alpha \in(0, \bar{\alpha}], \pi \succ \alpha f+(1-\alpha) \pi$.

Axiom A.7 (Weak Translation Invariance at Certainty) If $\succsim$ is locally EU at $\pi$ with respect to $p^{\pi}$ and locally EU at $\pi^{\prime}$ with respect to $p^{\pi^{\prime}}$ then $p^{\pi}=p^{\pi^{\prime}}$.

Axiom A.7 is very weak in the sense that it is trivially satisfied in the standard models, such as maxmin expected utility, variational preferences, confidence preferences, or the smooth model. Furthermore, it can be shown that axiom A.7 does not imply axiom A.5.

The main result of this section is the following theorem.

Theorem 3. Suppose there are at least three non-null states. The preference $\succsim$ satisfies axioms A.1-A.4, A.6, A.7, and P2 if and only if it is represented by

$$
f \mapsto \sum_{s \in S} \phi(u(f(s))) p(s)
$$

where $u: \Delta(Z) \rightarrow \mathbb{R}$ is a non-constant affine function, $p \in \Delta(S)$ such that $p(s)>0$ iff $s$ is non-null, and $\phi: \mathcal{U} \rightarrow \mathbb{R}$ is a continuous, increasing, and concave function. Moreover, in this case $p$ is the measure with respect to which $\succsim$ is Locally EU. Furthermore, if $(u, \phi, p)$ and $\left(u^{\prime}, \phi^{\prime}, p^{\prime}\right)$ represent $\succsim$, then there exists $\alpha, A>0$, $\beta, B \in \mathbb{R}$ such that $p^{\prime}=p, u^{\prime}=\alpha u+\beta$, and $\phi^{\prime}(\alpha r+\beta)=A \phi(r)+B$ for all $r$ in range of $u$. 
Remark 1. In the presence of axiom A.6 we only need to use a "one-sided" version of axiom A.7. Preferences are one-sided locally $E U$ at the constant act $\pi$ with respect to $p^{\pi} \in \Delta(S)$ if, for all acts $f: \mathbb{E}_{p^{\pi}} f \succ \pi$ implies there exists an $\bar{\alpha} \in(0,1]$ such that for all $\alpha \in(0, \bar{\alpha}], \alpha f+(1-\alpha) \pi \succ \pi$. The proof of Theorem 3 relies only on this property.

\section{Future work}

It would be desirable to obtain an axiomatization of SOEU without any concavity restrictions. The technical reason why we need axiom A.6 is that it guarantees the absolute continuity of functions $v_{s}$, which is needed to integrate the condition obtained from A.7, which is expressed in terms of derivatives of $v_{s}$.

In future versions of this paper we will explore conditions that guarantee absolute continuity, but do not impose concavity. Some known strengthenings of A.7, for example the Weak Certainty Independence axiom of Maccheroni et al. (2006) have this property, as they even imply Lipschitzianity. However, they are too strong for our purposes, as they restrict the functional form of $\phi$.

\section{A Proof of Theorem 1}

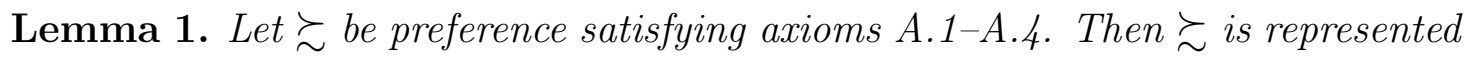
by $f \mapsto I(u(f))$ where $u: \Delta(Z) \rightarrow \mathbb{R}$ is affine with range $\mathcal{U}$ and $I: \mathcal{U}^{S} \rightarrow \mathbb{R}$ is normalized, continuous, and monotone on $\mathcal{U}^{S}$.

Proof. The proof of Lemma 67 of Cerreia-Vioglio, Maccheroni, Marinacci, and Montrucchio (2008) also applies to preferences that do not satisfy their Uncertainty Aversion axiom (witout delivering the quasiconcavity of $I$ ).

\section{Proof of Theorem 1}

The necessity of the axioms is routine. For sufficiency, note that the functional $I$ from Lemma 1 induces preferences $\succsim_{u}$ on $\mathcal{U}^{S}$ that are continuous, monotone, and satisfy P2 on utility acts. By Theorem 3 of Debreu (1960), there exist continuous 
functions $v_{s}: \mathcal{U} \rightarrow \mathbb{R}$ such that $\succsim_{u}$ is represented by

$$
V(u)=\sum_{s \in S} v_{s}(u(s))
$$

The function $u$ and at least three of the functions $v_{s}$ have to be non-constant in order to produce at least three non-null states.

\section{B Proof of Theorem 2}

\section{Proof of Theorem 2}

The necessity of the axioms and uniqueness of the representation are routine. For sufficiency, first, observe that by Lemma $1, \succsim$ is represented by $f \mapsto I(u(f))$ where $u: \Delta(Z) \rightarrow \mathbb{R}$ is affine with range $\mathcal{U}$ and $I: \mathcal{U}^{S} \rightarrow \mathbb{R}$ is normalized, continuous, and monotone on $\mathcal{U}^{S}$.

Second, show that $I$ satisfies Werner's (2005) "risk aversion" condition. GMambiguity aversion of $\succsim$ means that for all $\xi \in \mathcal{U}^{S}$ and $x \in \mathcal{U}: I(\xi) \geq x$ implies $\mathbb{E}_{p} \xi \geq x$. Fix $\xi \in \mathcal{U}^{S}$. Suppose that $\mathbb{E}_{p} \xi=\sup \mathcal{U}$. Then for all $s: \xi(s) \leq \mathbb{E}_{p} \xi$, so by monotonicity and normalization

$$
\mathbb{E}_{p} \xi \geq I(\xi)
$$

Suppose that $\mathbb{E}_{p} \xi<\sup \mathcal{U}$. For any sufficiently small $\varepsilon>0$ define $x_{\varepsilon}:=\mathbb{E}_{p} \xi+\varepsilon$. Then $x_{\varepsilon} \in \mathcal{U}$, so $x_{\varepsilon}>\mathbb{E}_{p} \xi$, so $x_{\varepsilon}>I(\xi)$, i.e., for all $\varepsilon>0$ : $\mathbb{E}_{p} \xi+\varepsilon>I(\xi)$. Thus for all $\xi \in \mathcal{U}^{S}$ inequality (2) holds.

Third, show that $p(s)>0$ iff $s$ is non-null. To see that suppose $p(s)=0$ and let $x, y \in \mathcal{U}$ be such that $I\left(x_{s} \xi\right)>I\left(y_{s} \xi\right)$. Let $\xi$ be a constant act equal to $y$. Then $I\left(x_{s} y\right)>I\left(y_{s} y\right)=I(y)=y=\mathbb{E}_{p}\left(x_{s} y\right)$, where the first inequality follows from P2 and the last equality from $p(s)=0$. This contradicts (2). The other direction is trivial.

Finally, by Werner's (2005) Theorem $1,{ }^{1}$ the preference on $\mathcal{U}^{S}$ induced by $I$ has a representation $\xi \mapsto \sum_{s \in S} \phi(\xi(s)) p(s)$ where $\phi: \mathcal{U} \rightarrow \mathbb{R}$ is a strictly increasing

\footnotetext{
${ }^{1}$ It can be verified that his theorem applies to preferences on $\mathcal{U}^{S}$ instead of just $\mathbb{R}^{S}$ and that his requirement that $I$ be strictly increasing can be dispensed with as long as there are at least three non-null states.
} 
and concave function. Moreover, $\phi$ is continuous as a consequence of the continuity of $I$.

\section{Proof of Theorem 3}

For any $\xi \in \mathbb{R}^{S}$ let $\|\xi\|:=\sum_{s \in S}|\xi(s)|$. Let $V:(\operatorname{int} \mathcal{U})^{S} \rightarrow \mathbb{R}$ be defined by $V(u):=\sum_{s} v_{s}(u(s))$ where $v_{s}: \operatorname{int} \mathcal{U} \rightarrow \mathbb{R}$ are weakly increasing and continuous functions. Let $D_{s}:=\left\{k \in \operatorname{int} \mathcal{U} \mid v_{s}^{\prime}(k)\right.$ exists $\}$ and let $D:=\cap_{s} D_{s}$.

Lemma 2. If $u \in(\operatorname{int} \mathcal{U})^{S}$ is such that $u_{s} \in D_{s}$ then $\nabla V$ exists at $u$ and is equal to the vector $\chi:=\left(v_{s}^{\prime}\left(u_{s}\right)\right)_{s}$.

Proof.

$$
\begin{aligned}
\frac{|V(u+v)-V(u)-\xi \cdot v|}{\|v\|} & =\frac{\left|\sum v_{s}\left(u_{s}+v_{s}\right)-\sum v_{s}\left(u_{s}\right)-\sum \chi_{s} v_{s}\right|}{\|v\|} \\
& =\left|\sum \frac{\left\{v_{s}\left(u_{s}+v_{s}\right)-v_{s}\left(u_{s}\right)-\chi_{s} v_{s}\right\}}{\|v\|}\right| \\
& \leq \sum \frac{\left|v_{s}\left(u_{s}+v_{s}\right)-v_{s}\left(u_{s}\right)-\chi_{s} v_{s}\right|}{\|v\|} \\
& \leq \sum \frac{\left|v_{s}\left(u_{s}+v_{s}\right)-v_{s}\left(u_{s}\right)-\chi_{s} v_{s}\right|}{\left|v_{s}\right|}
\end{aligned}
$$

and the limit of the RHS is zero as a consequence of differentiability of all functions $v_{s}$ at $u_{s}$.

Lemma 3. For all $\pi \in \Delta(Z)$ such that $u(\pi)=: k \in D$ and $\nabla V(k) \neq 0$ the preference represented by $V(u(f))$ is locally $E U$ at $\pi$ with respect to $p:=\frac{\nabla V(k)}{\|\nabla V(k)\|}$.

Proof. Suppose that $f \in \mathcal{F}$ is such that $p \cdot f \succ \pi$. Then $u(p \cdot f)>k$, so $p \cdot u(f)>$ $p \cdot k e$, so $p \cdot[u(f)-k e]>0$, so $\nabla V(k e) \cdot[u(f)-k e]>0$. Suppose toward contradiction that $(0,1) \ni \alpha_{n} \rightarrow 0$ such that $V\left(k e+\alpha_{n}(u(f)-k e)\right)-V(k e) \leq 0$. By differentiability of $\left.V, \frac{1}{\alpha_{n}}\left[V\left(k e+\alpha_{n}(u(f)-k e)\right)-V(k e)-\alpha_{n} \nabla V(k e) \cdot(u(f)-k e)\right)\right] \rightarrow$ 0, i.e., $\left.\frac{1}{\alpha_{n}}\left[V\left(k e+\alpha_{n}(u(f)-k e)\right)-V(k e)\right] \rightarrow \nabla V(k e) \cdot(u(f)-k e)\right)$. But the LHS $\leq 0$ and RHS $>0$, so contradiction. 
Proof of Theorem 3. The necessity of the axioms and uniqueness of the representation are routine. For sufficiency, observe that like in the proof of Theorem 1 $\succsim_{u}$ is represented by

$$
V(u)=\sum_{s \in S} v_{s}(u(s))
$$

where $v_{s}: \mathcal{U} \rightarrow \mathbb{R}$ are continuous, weakly increasing functions, at least three of them nonconstant. Consider the restriction of $\succsim_{u}$ to $\operatorname{int} \mathcal{U}^{S}$. Further, let $T \subset S$ indicate the nonconstant functions $v_{s}$; consider the restriction of $\succsim_{u}$ to int $\mathcal{U}^{T}$. By Theorem 3 of Debreu and Koopmans (1982) there exists $t \in T$ such that all functions $v_{s}$ for $s \neq t$ are concave; hence each of the sets $\operatorname{int} \mathcal{U}-D_{s}$ is at most countable. Moreover, their Theorem 3 also guarantees that int $\mathcal{U}-D_{t}$ is countable, and the derivative of $v_{t}$ is positive at those points. Thus, $D:=\cap_{s} D_{s} \subseteq \operatorname{int} \mathcal{U}$ is such that int $\mathcal{U}-D$ is at most countable and all functions $v_{s}$ are differentiable on $D$. Observe that by Lemma 2 for any $k \in D$ the derivative $\nabla V(k e)$ exists and is equal to the vector $\chi:=\left(v_{s}^{\prime}(k)\right)_{s \in S}$. Note that $\chi \neq 0$ because the derivative of $v_{t}$ is positive, as noted above.

By Lemma $3, \succsim$ is locally EU at any $\pi \in \Delta(Z)$ such that $u(\pi)=: k \in D$ with respect to $p^{k}=\frac{\left(v_{s}^{\prime}(k)\right)_{s \in S}}{\left\|\left(v_{s}^{\prime}(k)\right)_{s \in S}\right\|}$. By our Axiom A.7. $p^{k}$ is independent of $k$ for all $k \in D$. But this means that for any $s \in T$

$$
\frac{v_{s}^{\prime}(k)}{v_{1}^{\prime}(k)}=\frac{v_{s}^{\prime}\left(k^{\prime}\right)}{v_{1}^{\prime}\left(k^{\prime}\right)}
$$

for all $k, k^{\prime} \in D$ where we have wlog assumed that $1 \in T$. Thus, there exists a number $\alpha_{s}>0$ such that for all $k \in D$

$$
v_{s}^{\prime}(k)=\alpha_{s} v_{1}^{\prime}(k)
$$

Define $\Phi_{s}: \mathcal{U} \rightarrow \mathbb{R}$ by $\Phi(k):=v_{s}(k)-\alpha_{s} v_{1}(k)$. Observe that by (3) the function $\Phi_{s}$ is differentiable at all points of $D$ with derivative equal zero. This implies (see, e.g., Kuczma, 1985, p.74) that $\Phi_{s}(b)$ is constant on $\mathcal{U}$, i.e., there exists $Z_{s} \in \mathbb{R}$ such that $v_{s}(k)=\alpha_{s} v_{1}(k)+Z_{s}$. Let $\alpha_{1}:=1$ and $\alpha_{s}:=0$ for all $s \notin T$. Defining $\phi(k):=v_{1}(k)$ for all $k \in \mathcal{U}$ and $p(s):=\frac{\alpha_{s}}{\sum_{s \in S} \alpha_{s}}$ for all $s \in S$ concludes the proof. 


\section{References}

Abdellaoui, M., A. Baillon, L. Placido, and P. P. Wakker (2009): "The Rich Domain of Uncertainty," mimeo.

Cerreia-Vioglio, S., F. Maccheroni, M. Marinacci, and L. MontrucCHIO (2008): "Uncertainty Averse Preferences," mimeo.

Chateauneuf, A. And J. H. Faro (2009): "Ambiguity through Confidence Functions," Journal of Mathematical Economics.

Chew, S. H. And J. S. SAgi (2008): "Small Worlds: Modeling Attitudes towards Sources of Uncertainty," Journal of Economic Theory, 139, 1-24.

Debreu, G. (1960): "Topological methods in cardinal utility theory," in Mathematical methods in the social sciences, ed. by K. Arrow, S. Karlin, and P. Suppes, Stanford University Press, Stanford.

Debreu, G. And T. Koopmans (1982): "Additively decomposed quasiconvex functions," Mathematical Programming, 24, 1-38.

Ergin, H. And F. Gul (2009): "A Theory of Subjective Compound Lotteries," Journal of Economic Theory, 144, 899-929.

Ghirardato, P. And M. Marinacci (2002): "Ambiguity Made Precise: A Comparative Foundation," Journal of Economic Theory, 102, 251-289.

GilboA, I. AND D. Schmeidler (1989): "Maxmin expected utility with nonunique prior," Journal of Mathematical Economics, 18, 141-153.

Hansen, L. P. And T. J. Sargent (2001): "Robust Control and Model Uncertainty," The American Economic Review, 91, 60-66.

Klibanoff, P., M. Marinacci, And S. MukerJi (2005): "A smooth model of decision making under ambiguity," Econometrica, 73, 1849-1892.

Kuczma, M. (1985): An Introduction to the Theory of Functional Equations and Inequalities., PWN, Kraków.

Maccheroni, F., M. Marinacci, And A. Rustichini (2006): "Ambiguity Aversion, Robustness, and the Variational Representation of Preferences," Econometrica, 74, 1447 - 1498. 
NAU, R. (2001): "Uncertainty Aversion with Second-Order Utilities and Probabilities," in Proceedings of the Second International Symposium on Imprecise Probabilities and Their Applications, http://www.sipta.org/isipta01/proceedings/063.html.

(2006): "Uncertainty Aversion with Second-Order Utilities and Probabilities," Management Science, 52, 136-145.

NeILson, W. S. (1993): "Ambiguity Aversion: An Axiomatic Approach Using Second Order Probabilities," mimeo.

[ (2009): "A Simplified Axiomatic Approach to Ambiguity Aversion," mimeo.

Rigotti, L., C. Shannon, and T. Strzalecki (2008): "Subjective Beliefs and Ex-Ante Trade," Econometrica, 76, 1167-1190.

Schmeidler, D. (1989): "Subjective Probability and Expected Utility without Additivity," Econometrica, 57, 571-587.

Strzalecki, T. (2009): "Axiomatic Foundations of Multiplier Preferences," mimeo.

WERNER, J. (2005): "A simple axiomatization of risk-averse expected utility," Economics Letters, 88, 73-77. 